\title{
The Burden of Smoking on the Financing of Healthcare
}

\author{
RIGELSKÝ, M. ${ }^{1}$, IVANKOVÁ, V. ${ }^{1}$, GONOS, J. ${ }^{1}$, GAVUROVÁ, B. ${ }^{2}$
}

1 | University of Prešov, Faculty of Management, Prešov, Slovak Republic

2) Charles University, First Faculty of Medicine, and General University Hospital in Prague, Department of Addictology, Prague, Czech Republic
Citation | Rigelský, M., Ivanková, V., Gonos, J., Gavurová, B. (2019). The Burden of Smoking on the Financing of Healthcare. Adiktologie, 19(2), 59-66; https://doi.org/10.35198/01-2019-002-0001
BACKGROUND: Smoking seems to be one of the serious health problems of present-day society, even in developed countries. In addition, smoking and its consequences may have a significant impact on health expenditure. AIM: Objective of the study is to determine the impact of smoking on health expenditure in a sample of the OECD member countries. DESIGN AND MEASUREMENTS (METHODS): Analytical procedures examine data from 2011 to 2018. Six variables, including two dependent (Health expenditure in US Dollars per capita and Health expenditure (\% of GDP)) and four independent variables focused on smoking and tobacco consumption.
SAMPLE: The sample consisted of all the OECD countries. RESULTS: For the variable identifying healthcare funding in terms of GDP, a impact was evident in smokers over 15 years of age, as well as those in the age range from 15 to 24 years. In the case of health expenditure per capita, an impact appeared only in smokers over 15 years of age. A medium-to-substantial rate of relation was identified in the link between health expenditure per capita and both smokers aged over 15 and smokers aged between 15 and 24. The relations that were analysed were negative. CONCLUSIONS: The results of the present study show that smoking has a negative impact on health expenditure.

\section{Keywords | Smoking Addiction - Smokers - Healthcare Expenditure - OECD}




\section{INTRODUCTION}

Healthcare is an integral part of everyday life, whether individual health or the health of the whole population is being discussed. The aim of each economy should be to improve the health status of the population and to extend the years of a productive and quality life free of disease and other health barriers. It is important to realize that the health of a population depends not only on the development of healthcare, but also on the involvement of the population and the strengthening of their control over the conditions that people may influence themselves. Many authors have agreed that health is a precondition for economic development and prosperity (European Commission, 2013), i.e. an important economic value, as the good health status of a population affects the economic growth of countries, including productivity, human capital, real income, and public spending (Bloom et al., 2004; Boachie, 2017; Sharma 2018). Consequently, poor health is associated with the reduction of human potential and depletion of public resources. With regard to smoking, many studies have dealt with the impact of smoking on health (Adda \& Lechene, 2013; Carter et al., 2015; Lopez-Nicolas et al., 2018). Also, Darden et al. (2018) stated that smoking is detrimental to health and causes morbidity and mortality. Despite the fact that the prevalence of smoking has declined over time and across age cohorts (Di Novi \& Marenzi, 2019), it seems to be one of the serious health problems of contemporary society, even in developed countries. Looking to the future, tobacco consumption in the Czech Republic will grow until 2028; the projected number of smokers in 2028 is 4-8\% higher than in 2013 (Janda \& Strobl, 2019). Smoking places tremendous financial and health burdens upon both society and individuals (Chung et al., 2007). The economic costs of tobacco use are substantial and include significant healthcare costs for treating the disease caused by tobacco use, as well as the lost human capital that results from the morbidity and mortality attributable to tobacco (WHO, 2019). Smoking and its consequences may have a significant impact on healthcare expenditure. The present study focuses on determining the impact of smoking on health expenditure in a sample of OECD countries.

\section{THEORETICAL BACKGROUND}

The financing of healthcare is one of the main elements of each economy, and its return may be multiplied in the form of a healthy and productive population. Health is a significant part of public budgets. This is demonstrated by the very rapidly increasing trend and the assumptions that by 2060 public health expenditure will have increased by one third (EC, 2013). While health expenditure was around 5.8\% of GDP in 1990, it was $8.7 \%$ in 2015 and it is projected to rise to $12.6 \%$ of GDP by 2060 (OECD, 2015; 2016a). Looking at the year-on-year comparison of health expenditure in the OECD countries between 2010 and 2018, there is an increase of 29.6\% (OECD, 2019a). Toader et al. (2017) confirmed that the health status of the population is primarily dependent on the financing of healthcare and consumption habits. Unhealthy consumption reduces health and longevity and this fact should be taken into account by individuals contemplating the consumption of addictive goods (Strulik, 2018). The decisions to start or quit smoking are different for each individual, but an important aspect to consider is information about the health risk of smoking (Lillard \& Onder, 2019). As the evidence shows, the current smoking status among people differs according to gender, ethnicity, religion, and household income. Conto et al. (2019) revealed that men are more inclined to smoke than women are, and smoking decreases as education and household income increase. Muhyedin et al. (2019) also identified a significant difference between males and females, as well as concluding that smoking habits are influenced by religion. Keto et al. (2017) confirmed that smokers use primary healthcare more frequently per year than non-smokers. Additionally, Li et al. (2018) found similar results in their study. Keto et al. (2017) adds that this phenomenon not only predicts an elevated incidence of serious illnesses, but it also imposes an economic burden on the healthcare system. According to the WHO $(2013,2019)$, tobacco kills more than 8 million people a year around the world and it causes more than half a trillion dollars' worth of economic damage each year. More than 7 million of those deaths are a result of direct tobacco use, while around 1.2 million are a result of non-smokers being exposed to second-hand smoke. Smoking is responsible not only for deaths, but also for acute myocardial infarctions, strokes, cancer diagnoses, and millions of years of potential life lost. Therefore, Pinto et al. (2019) proposed that taxes on cigarettes should be increased in order to reduce morbidity and mortality. Smoking represents a heavy economic burden all over the world. The level of healthcare expenditure caused by diseases attributable to smoking was $5.7 \%$ of global health expenditure in 2012 (Goodchild et al., 2018). Ekpu and Brown (2015) state that the economic burden of smoking represents about $15 \%$ of the aggregate healthcare expenditure in high-income countries. On the other hand, the economic activities generated by the production and consumption of tobacco provide an economic stimulus, such as tax revenues, employment, or cost savings in pension payments from the premature deaths of smokers. The authors agree, in their conclusion, that while tobacco smoking may be economically beneficial, its direct costs to society far outweigh any benefits. As Alcaraz et al. (2016) argue, tobacco smoking accounts for a significant proportion of healthcare expenditure in Latin America, and the tax collected from cigarette sales is far from covering this. The results of their study show that the cost attributed to tobacco smoking ranged from $5.2 \%$ to $12.7 \%$ of healthcare expenditure, and tax collection from cigarette sales covers almost 35\% of the healthcare expenditure attributed to tobacco smoking. There are several studies that deal with the relationship between healthcare expenditure and smoking (Harrison et al., 2003). Most authors agree that the total economic loss from smoking-related diseases highlights the significant loss to society, the health sector, and the country's economy (Bundhamcharoen et al., 2016). Hayashida et al. (2010) confirmed that smokers have a higher mortality rate, shorter life expectancy, and generally higher annual medical expenditure than non-smokers. The results of 
other studies show that current smokers have higher health costs than former smokers (Barnett et al., 2015, Maciosek et al., 2015). Generally, it is believed that reducing the prevalence of smoking has a positive effect on increasing public resources as a result of the reduction in healthcare expenditure related to the treatment of poor health caused by smoking. Nevertheless, several authors argue that smokers have no significant impact on healthcare expenditure in the long run compared to non-smokers. This statement is based on the fact that the increased healthcare expenditure of smokers over their lifetime is balanced by their reduced life expectancy (Leu \& Schaub, 1984). Consequently, smokers do not require any additional old-age healthcare expenditure compared to non-smokers. On the other hand, healthcare expenditure and the loss of economic output resulting from premature death should be taken into account (Owen et al., 2019). Kontsevaya and Kalinina (2011) analysed the costs of healthcare in Russia. They confirmed that smoking is associated with significant healthcare expenditure and highlighted investment in preventive programmes to reduce the prevalence of smoking. Izumi et al. (2001) also state that smokers consume excess medical care, and Max (2001) adds that this leads to increased healthcare expenditure over their lifetime. Lightwood and Glantz (2016) conducted a study that dealt with smoking behaviour and healthcare expenditure in the United States (1992-2009) and the results showed that the states with lower rates of smoking have substantially lower medical costs and those that have higher rates of smoking have higher medical costs. Higher health expenditure may be associated with ambulatory health services (Miller et al. 1999), but also with smoking-related hospitalizations (Tai et al., 2018). Thus, smoking still imposes a substantial economic burden in terms of total costs (Chen et al., 2019), and in their conclusions many authors have highlighted the need for a more stringent tobacco control strategy to reduce smoking (Polakowska et al., 2017; Xu et al., 2015; Yang et al., 2011). There is evidence of health-promoting interventions that target proximal behavioural risk factors, such as tobacco having important effects on health outcomes (Cylus et al. 2018). A reduction of smoking is also significant for changes in healthcare expenditure; it is expected that a decrease in smoking will be followed by a reduction in healthcare expenditure (Lightwood \& Glantz, 2016). Barendregt et al. (1997) outlined the relation between smoking and healthcare costs and confirmed that smoking cessation leads to savings in healthcare costs, but only in the short term. Conversely, quitting smoking would lead to increased healthcare costs in the long term. According to the OECD (2016b), the trend in the OECD countries is a negative percentage change in smoking, as evidenced by an average decrease in smoking by $24.4 \%$ between 2000 and 2013; however, smoking is one of the biggest public health threats and the impact of smoking on healthcare expenditure needs to be addressed.

\section{METHODOLOGY}

The primary objective of the study is to determine the impact of smoking on health expenditure in a sample of OECD countries. The objective will be met by analytical procedures that examine data from 2011 to 2018. Six variables enter the processing; two dependent variables are represented: H_EXP_US\$ - Health expenditure in US Dollars per capita and H_EXP_\% - Health expenditure (\% of GDP), and four independent variables are represented: Smors_D\%15 - Daily smokers in \% of population 15+, Smors_D\%15-24 - Daily smokers in \% of population 15-24, Cig_D - Number of cigarettes per day per capita, G_Tab - Grams yearly per capita. From the point of view of the correct methodological approach, the following research question and two main hypotheses were formulated:

\section{RQ: Is there any impact of smoking on health expenditure?}

H1: It is assumed that there is a significant impact of selected smoking-related characteristics on health expenditure as a percentage of GDP in the OECD countries.

$\mathrm{H} 2$ : It is assumed that there is a significant impact of selected smoking-related characteristics on health expenditure in US Dollars per capita in the OECD countries.

The data that was analysed (variables) was collected from the OECD databases (OECD, 2019b). H_EXP_US\$ - Health expenditure in US Dollars per capita represents the total health expenditure of all healthcare providers in all fund schemes expressed in US dollars. H_EXP_\% - Health expenditure (\% of GDP) represents the total health expenditure of all healthcare providers in all fund schemes expressed as a percentage of gross domestic product (GDP). The independent variables include Smors_D\%15 - Daily smokers in \% of population $15+$, which expresses the ratio of smokers over the age of 15 to the total population of a given state; Smors_D\%15-24 - Daily smokers in \% of population 15-24, which expresses the ratio of smokers over 15 and under 24 years (inclusive) to the total population of a given state; Cig_D - the number of cigarettes per day per capita, expressing the average number of cigarettes consumed per person per day, without age specification; G_Tab - Grams yearly per capita, which expresses the average tobacco consumption in grams per person older than 15 years (inclusive). The above data was collected from 2011 to 2018 for all OECD countries (36).

A method of regression analysis, multiple linear regression (OLS), was used for statistical processing. On the basis of the Gauss-Markov theorem, the primary focus was on the homogeneity (constancy) of variability of residues (homoscedasticity) and multicollinearity, which are the two most significant characteristics influencing the BLUE (best linear unbiased estimator) estimate. Heteroscedasticity was tested by using the Breusch-Pagan test (Breusch \& Pagan, 1979) and multicollinearity by using VIF (Variance Inflation Factors) statistics (Fox \& Monette, 1992). Outliers were tested by using the Bonferroni test (Cook \& Weisberg, 1982). A correlation analysis was also displayed. Multivariate normality was tested with the help of Henze-Zirkler's multivariate normality test (Henze \& Zirkler, 1990). On the basis of this output, the Spearman coefficient $\rho$ was chosen as an 
alternative to the parametric Pearson $\mathrm{r}$. The analytical processes were realized by using the programming language R v 3.6.1 (Action of the Toes).

\section{RESULTS}

The next section of the study is devoted to analytical processing leading to the fulfilment of the assumptions and objectives stated in the previous section. This section is divided into the basic output of descriptive statistics and three separate parts. The first part is devoted to the processes leading to the verification of the first main hypothesis; the second part includes the processes related to the second hypothesis, and the last part represents the correlation analysis.

Table 1 shows the basic descriptive statistics used to complete the research idea and identify the variables that were examined.

The first rows of Table 1 show the amount of data that entered the processing, as well as the amount of missing data. As may be deduced, many observations are missing. Therefore, data from earlier years was also used. It was eight years ago that the occurrence of missing values was very frequent. In this case, specific characteristics such as stationarity or autocorrelation are not a threat that could affect the overall output of the model. When assessing the central tendencies of the variables being analysed, it is appropriate to focus on the rate itself on the one hand, and on the other hand, on the difference between the mean and median, where excessive deviations indicate a non-compli- ance with the normal distribution. The standard deviation identifies the rate of fluctuation from the average. Skewness and kurtosis are identifiers that assess deviations from the normal distribution, and exceeding the interval from -1 to 1 indicates discrepancy. Quartiles indicate a rate of $25 \%$ of the lowest and $75 \%$ of the highest values.

\subsection{Impact of smoking on health expenditure as a percentage of GDP}

On the basis of the Gauss-Mark theorem, it is necessary to verify the conditions of multicollinearity and heteroscedasticity. The VIF outputs are less than five for all independent variables (Smors_D\%15 - 3.7048; Smors_D\%15-24 3.100; G_Tab - 1.5050; Cig_D - 1.9199). Therefore, the rate of multicollinearity is acceptable. Homoscedasticity was verified by using the Breusch-Pagan test, which has a pvalue of 0.01428 for a BP statistic of 12.453 and four degrees of freedom. This value is less than 0.05. Thus, there are significant deviations in the homogeneity of variability of residues, i.e. there is heteroscedasticity. The HC3 estimator was used to derive the significance of the impact. The Bonferroni outlier test does not show significant outliers. Table 2 (below) shows the outputs of the regression model of this part of the analysis.

The most important information in Table 2 is the p-value, shown in the last column. As may be seen, this value is less than 0.05 for Smors_D\%15 and Smors_D\%15-24. At the $\alpha$ level of 0.1 , the Cig_D variable may also be considered as an indicator that has a significant impact on health expenditure. It may not be considered that G_Tab has a sig-

\begin{tabular}{lcccccc}
\hline Descriptive & H_EXP_\% & H_EXP_US\$ & Smors_D\%15 & Smors_D\%15-24 & G_Tab & Cig_D \\
\hline $\mathrm{N}$ & 288 & 288 & 144 & 129 & 143 & 117 \\
\hline missing N & 0 & 0 & 144 & 17.1431 & 15.0535 & 1324.4091 \\
\hline Mean & 8.7720 & 3559.9826 & 17.0500 & 14.8000 & 1120.1000 & 13.0957 \\
\hline Median & 8.9330 & 3483.5000 & 4.2979 & 5.8911 & 583.7598 & 2.3247 \\
\hline Std. Deviation & 2.3167 & 1740.2646 & 0.1173 & 0.3292 & 1.3983 & -0.1723 \\
\hline Skewness & 0.7678 & 0.9711 & -0.4315 & 0.2756 & 2.0613 & 0.6305 \\
\hline Kurtosis & 1.8980 & 1.7971 & 13.8500 & 11.5000 & 905.0000 & 11.5000 \\
\hline Q1 & 7.0228 & 2123.7500 & 19.9750 & 18.5000 & 1625.0000 & 14.7000 \\
\hline Q3 & 10.3190 & 4697.0000 & & & &
\end{tabular}

Table 1 | Descriptive statistics of variables

\begin{tabular}{lcccc}
\hline OLS model HC3 & Estimate & Std.Error & t value & Pr $(>|\mathbf{t}|)$ \\
\hline (Intercept) & 13.9937 & 2.1686 & 6.4529 & $2.13 \times 10^{-8}$ \\
\hline Smors_D\%15 & -0.7988 & 0.1717 & -4.6513 & $1.86 \times 10^{-5}$ \\
\hline Smors_D\%15-24 & 0.1762 & 0.0692 & 2.5468 & $1.35 \times 10^{-2}$ \\
\hline G_Tab & 0.0005 & 0.0005 & 1.0282 & $3.08 \times 10^{-1}$ \\
\hline Cig_D & 0.4659 & 0.2663 & 1.7494 & $8.53 \times 10^{-2}$ \\
\hline
\end{tabular}

Table 2 | Regression model - OLS HC3 (y = H_EXP_\%) 


\begin{tabular}{lcccc}
\hline OLS model & Estimate & Std.Error & $\mathbf{t}$ value & $\operatorname{Pr}(>|\mathbf{t}|)$ \\
\hline (Intercept) & 8861.8228 & 1383.9007 & 6.4040 & $2.58 \times 10^{-8}$ \\
\hline Smors_D\%15 & -491.4469 & 85.0567 & -5.7780 & $2.88 \times 10^{-7}$ \\
\hline Smors_D\%15-24 & 22.6550 & 49.3351 & 0.4590 & $6.48 \times 10^{-1}$ \\
\hline G_Tab & 0.4179 & 0.3915 & 1.0670 & $2.90 \times 10^{-1}$ \\
\hline Cig_D & 240.6734 & 128.4714 & 1.8730 & $6.59 \times 10^{-2}$ \\
\hline
\end{tabular}

Table 3 | Regression model - OLS (y = H_EXP_US\$)

\begin{tabular}{lcccccccc}
\hline \multirow{2}{*}{ hz mvn test } & \multicolumn{2}{c}{ Smors_D\%15 } & \multicolumn{2}{c}{ Smors_D\%15-24 } & \multicolumn{2}{c}{ G_Tab } & \multicolumn{2}{c}{ Cig_D } \\
\cline { 2 - 10 } & $\mathbf{h z}$ & $\mathbf{p}$ value & hz & $\mathbf{p}$ value & hz & p value & hz & p value \\
\hline H_EXP_\% & 1.7508 & 0.0005 & 1.7324 & 0.0005 & 5.5804 & 0.0000 & 3.0858 & 0.0000 \\
\hline H_EXP_US\$ & 2.3651 & 0.0000 & 2.0071 & 0.0000 & 4.9452 & 0.0000 & 1.9032 & 0.0002 \\
\hline
\end{tabular}

Table 4 | Multivariate normality test

\begin{tabular}{|c|c|c|c|c|c|}
\hline relationship $\rho$ & & Smors_D\%15 & Smors_D\%15-24 & G_Tab & Cig_D \\
\hline \multirow[t]{3}{*}{$\overline{H \_E X P \_\%}$} & $\rho$ & -0.267 & -0.213 & $x$ & $x$ \\
\hline & $p$ value & 0.001 & 0.016 & 0.130 & 0.845 \\
\hline & $\mathrm{N}$ & 144 & 129 & 143 & 117 \\
\hline \multirow[t]{3}{*}{ H_EXP_US\$ } & $\rho$ & -0.471 & -0.494 & -0.262 & $x$ \\
\hline & $p$ value & 0.000 & 0.000 & 0.002 & 0.678 \\
\hline & $\mathrm{N}$ & 144 & 129 & 143 & 117 \\
\hline
\end{tabular}

Table 5 | Relationship analysis - Spearman $\rho$

nificant impact on health expenditure. Consequently, the hypothesis H1 (It is assumed that there is a significant impact of selected smoking-related characteristics on health expenditure as a percentage of GDP in the OECD countries) may be confirmed. For the F-statistic of 15.93, the model obtained a value of Multiple R-squared: 0.5151 and Adjusted R-squared: 0.4828. These values are acceptable.

\subsection{Impact of smoking on health expenditure in US Dollars per capita}

In the previous section, multicollinearity was verified and its measured rate was considered acceptable. Homoscedasticity was verified by using the Breusch-Pagan test, which has a p-value of 0.015 for a BP statistic of 6.7454 and four degrees of freedom. This value is higher than 0.05 , and thus the assumption of homoscedasticity is met. The Bonferroni outlier shows significant outliers. The Bonferroni outlier test shows the presence of one outlier $(p$-value $=0.13234)$, but this outlier appears to be acceptable. Table 3 shows the outputs of the regression model of this part of the analysis.

The most important information in Table 3 is the p-value, shown in the last column. As is evident, this value is less than 0.05 only for Smors_D\%15. At the $\alpha$ level of 0.1, the Cig_D variable may also be considered as an indicator that has a significant impact on health expenditure. It may not be considered that G_Tab and Smors_D\%15-24 have a significant impact on health expenditure. Thus, the hypothesis $\mathrm{H} 2$ (It is assumed that there is a significant impact of selected smoking-related characteristics on health expenditure in US Dollars per capita in the OECD countries) may be confirmed. For the F-statistic of 20.55, the model obtained a value of Multiple R-squared: 0.5781 and Adjusted R-squared: 0.55. These values are acceptable.

\subsection{Relation between health expenditure and smoking}

The next part points to the relations between the individual variables representing health expenditure and selected variables related to smoking. One of the basic conditions for the application of the parametric test (Pearson's $r$ ) is the approximate normality of the data being analysed. Table 4 shows the outputs of Henze-Zirkler's multivariate normality test.

On the basis of the fact that the p-value is not higher than 0.05 in any case, the condition of normality was not met. Table 5 shows the output of a non-parametric Spearman test of relation.

When interpreting Table 5, it is appropriate, in the first step, to focus on the $\mathrm{p}$-value that is higher than 0.05 in three re- 
lationships. Thus, there is no significant relation. Analysis of the relationships also points to a low-to-medium rate of relation in the case of the link between H_EXP_\% and both Smors_D\%15 and Smors_D\%15-24. For the link between $\mathrm{H}_{-}$ EXP_US\$ and the G_Tab variable, there is also a low-to-medium rate of relation. A medium-to-substantial rate of relation may be identified in the link between H_EXP_US $\$$ and both Smors_D\%15 and Smors_D\%15-24. In all of the above-mentioned relationships, there is a negative rate of the $\rho$ coefficient and the negative relation is confirmed.

\section{CONCLUSION AND DISCUSSION}

As mentioned in the introduction, smoking is the cause of many diseases and much mortality (Darden et al., 2018) and a negative impact on health expenditure may be expected. Strulik (2018) also stated that the consumption of addictive goods generally affects consumer health. As a consequence of this, it may be predicted that the increased consumption of addictive goods leads to an increased burden on the financing of healthcare. On the other hand, there are some controversies over the economic effect of smoking when one looks at the economic consequences of smoking in a wider perspective. These controversies deal with the life expectancy of smokers (lower than non-smokers), tax revenues, employment, or cost savings in pension payments from the premature death of smokers (Leu \& Schaub, 1984, Ekpu \& Brown, 2015). However, the main focus of this study was only on the expenditure side. The primary objective of the study is to determine the impact of smoking on health expenditure in a sample of OECD countries. The objective was met by three steps of analytical procedures that examine data from 2011 to 2018. In the first step, the impact of selected smoking-related indicators on health expenditure as a percentage of GDP was deduced. In the second step, the impact of selected smoking-related indicators on health expenditure in US Dollars per capita was analysed. In the third step, an analysis of the relationships between smoking-related indicators and health expenditure was realized. For the first two steps above, two main hypotheses were formulated (H1: It is assumed that there is a significant impact of selected smoking-related characteristics on health expenditure as a percentage of GDP in the OECD countries; H2: It is assumed that there is a significant impact of selected smoking-related characteristics on health expenditure in US Dollars per capita in the OECD countries) and the third step identified the picture of the relations. Both hypotheses were confirmed. For the variable identifying healthcare funding in terms of GDP, a causal relation was evident in smokers over 15 years of age, as well as for the age range from 15 to 24 years. In the case of health expenditure per capita, a causal relation appeared only in smokers over 15 years of age. Analysis of the relationships points to a low-to-medium rate of relation in the case of the link between health expenditure as a percentage of GDP and both smokers aged over 15 and smokers aged between 15 and 24. For the link between health expenditure per capita and grams yearly per capita, there was also a low-to-medium rate of relation. A medium-to-substantial rate of relation was identified in the link between health expenditure per capita and both smokers aged over 15 and smokers aged between 15 and 24. As predicted, the relations that were analysed were negative. These findings confirm the statements of many authors (Izumi et al., 2001; Max, 2001; Hayashida et al., 2010; Kontsevaya \& Kalinina, 2011). Keto et al. (2017) and Li et al. (2018) also point to an increased frequency of primary healthcare use among smokers in comparison to non-smokers, leading to increased health expenditure. By analogy, being a smoker is a stronger predictor of healthcare consumption. Lightwood and Glantz (2016) highlighted the fact that states with higher rates of smoking have higher health expenditure than states where smoking is less frequent. The negative consequences of smoking are therefore evident in terms of health expenditure.

From an applied point of view, negative relations may be interpreted as meaning that if the proportion of smokers or another statistically significant indicator decreases, health expenditure will decrease. At this point, the need for prevention leading to a reduction in the number of smokers in the population should be highlighted. The tools currently in use appear to be ineffective in many countries. The impact and relations confirmed in this study may be considered secondary. It may be assumed that smoking-related diseases, not smoking itself, are the cause of an increase in health expenditure, and this is a limitation of the research. Future research will focus on determining the primary consequences of smoking (COPD) for health expenditure. It is also planned to work with a longer time series as well as to enrich the relations with mediating variables e.g. the funding system. An assessment of the effectiveness of the preventive tools related to a reduction of smoking in individual countries is also considered necessary in future research.
Authors' contribution: Conceptualisation: Beáta Gavurová; Data curation: Martin Rigelský; Methodology: Jaroslav Gonos and Viera Ivanková; Formal analysis: Martin Rigelský; Results: Beáta Gavurová and Viera Ivanková; Visualisation: Martin Rigelský; Final edition: Viera Ivanková and Beáta Gavurová. All authors have read and agreed to the published version of the manuscript.
Declaration of interest: Authors declare that they do not have any competing financial, professional, or personal interests from other parties. 


\section{REFERENCES}

Adda, J., Lechene, V. (2013). Health Selection and the Effect of Smoking on Mortality. Scandinavian Journal of Economics, 115(3), 902-931. doi: $10.1111 /$ sjoe. 12004 .

Alcaraz, A., Pichon-Riviere, A., Bardach, A., Augustovski, F. (2016). The Economic Impact of Tobacco Smoking In The Latin-American Healthcare Systems: A Study Conducted In Seven Countries And Its Extrapolation At The Regional Level. Value in Health, 197), A628. doi: 10.1016/j.jval.2016.09.1618.

Barendregt, J. J., Bonneux, L., van der Maas, P. J. (1997). The health care costs of smoking. New England Journal of Medicine, 337(15), 1052-1057. doi: 10.1056/NEJM199710093371506.

Barnett, P. G., Hamlett-Berry, K., Sung, H., Max, W. (2015). Health Care Expenditures Attributable to Smoking in Military Veterans. Nicotine \& Tobacco Research, 17(5), 586-591. doi: 10.1093/ntr/ntu187.

Bloom, D. E., Canning, D., Sevilla, J. (2004). The effect of health on economic growth: A production function approach. World Development, 32(1), 1-13.

Boachie, M. K. (2017). Health and Economic Growth in Ghana: An Empirical Investigation. Fudan Journal of the Humanities and Social Sciences, 102), 253-265. doi: 10.1007/s40647-016-0159-2.

Breusch, T. S., Pagan, A. R. (1979). A Simple Test for Heteroscedasticity and Random Coefficient Variation. Econometrica, 47, 1287-1294.

Bundhamcharoen, K., Aungkulanon, S., Makka, N., Shibuya, K. (2016). Economic burden from smoking-related diseases in Thailand. Tobacco Control, 25(5), 532-537. doi: 10.1136/tobaccocontrol-2015-052319.

Carter, B. D., Abnet, C. C., Feskanich, D., Freedman, N. D., Hartge, P., Lewis, C. E., Ockene, J. K., Prentice, R. L., Speizer, F. E., Thun, M. J., Jacobs, E. J. (2015) Smoking and Mortality - Beyond Established Causes. New England Journal of Medicine, 372(7), 631-640. doi: 10.1056/NEJMsa1407211.

Chen, J., McGhee, S., Lam, T. H. (2019). Economic Costs Attributable to Smoking in Hong Kong in 2011: A Possible Increase From 1998. Nicotine \& Tobacco Research, 21(4), 505-512. doi: 10.1093/ntr/ntx254.

Chung, C., Wang, J., Yu, C., Yang, M. (2007). Lifetime medical expenditure and life expectancy lost attributable to smoking through major smoking related diseases in Taiwan. Tobacco Control, 16(6), 394-399. doi: 10.1136/tc.2006.018986.

Conto, R. M., Paullin, M., Stephenson, J. (2019). Descriptive Epidemiology of Smoking Status in a Real World Sample of Adults in the United States: 2010-2017. Value in Health, 22, S297. doi: 10.1016/j.jval.2019.04.1424.

Cook, R. D., Weisberg, S. (1982). Residuals and Influence in Regression. New York: Chapman and Hall. Available at: https://conservancy.umn.edu/ handle/11299/37076.

Cylus J., Permanand, G., Smith, P.C. (2018). Making the economic case for investing in health systems. What is the evidence that health systems advance economic and fiscal objectives? Copenhagen (Denmark): European Observatory Policy Brief Series. Available at: http://www.euro.who.int/__data/assets/ pdf_file/0010/380728/pb-tallinn-01-eng.pdf?ua=1.

Darden, M., Gilleskie, D. B., Strumpf, K. (2018). Smoking and Mortality: New Evidence from a Long Panel. International Economic Review, 59(3), 1571-1619. doi: 10.1111/iere.12314.

Di Novi, C., Marenzi, A. (2019). The smoking epidemic across generations, genders, and educational groups: A matter of diffusion of innovations. Economics \& Human Biology, 33, 155-168. doi: 10.1016/j.ehb.2019.01.003.

Ekpu, V. U., Brown, A. K. (2015). The Economic Impact of Smoking and of Reducing Smoking Prevalence: Review of Evidence. Tobacco Use Insights, 8 , 1-35. doi: 10.4137/TUI.S15628.

European Commission. (2013). Commission staff working document - Investing in Health. Available at: https://ec.europa.eu/health/sites/health/files/policies/ docs/swd_investing_in_health.pdf.

Fox, J., Monette, G. (1992). Generalized collinearity diagnostics. JASA, 87, 178-183.
Goodchild, M., Nargis, N., d'Espaignet, E. T. (2018). Global economic cost of smoking-attributable diseases. Tobacco Control, 27(1), 58-64. doi: 10.1136/tobaccocontrol-2016-053305

Harrison, G. W., Feehan, J. P., Edwards, A. C., Segovia, J. (2003). Cigarette smoking and the cost of hospital and physician care. Canadian Public PolicyAnalyse De Politiques, 29(1), 1-20.

Hayashida, K., Imanaka, Y., Murakami, G., Takahashi, Y., Nagai, M., Kuriyama, S., Tsuji, I. (2010). Difference in lifetime medical expenditures between male smokers and non-smokers. Health Policy, 94(1), 84-89. doi: 10.1016/j.healthpol.2009.08.007.

Henze, N., Zirkler, B. (1990). A Class of Invariant Consistent Tests for Multivariate Normality. Communication in Statistics-Theory and Methods, 19(10), 35953618.

Izumi, Y., Tsuji, I., Ohkubo, T., Kuwahara, A., Nishino, Y., Hisamichi, S. (2001). Impact of smoking habit on medical care use and its costs: a prospective observation of National Health Insurance beneficiaries in Japan. International Journal of Epidemiology, 303), 616-621. doi: 10.1093/ije/30.3.616.

Janda, K., Strobl, M. (2019). Smoking Czechs: Modelling Tobacco Consumption and Taxation. Prague Economic Papers, 28(1), 3-29. doi: 10.18267/j.pep.685.

Keto, J., Ventola, H., Jokelainen, J., Timonen, M., Linden, K., Ylisaukko-oja, T., Keinanen-Kiukaanniemi, S., Auvinen, J. (2017). Primary health care utilisation and its costs among middle-aged smokers. European Journal of Health Economics, 18(3), 351-360. doi: 10.1007/s10198-016-0793-2.

Kontsevaya, A. V., Kalinina, A. M. (2011). Costs of the health care in Russia associated with smoking. Rational Pharmacotherapy in Cardiology, 7(3), 306-312.

Leu, R. F., Schaub, T. (1984). Economic aspects of smoking. Effective Health Care, 2(3), 111-123.

Li, C., Fan, Y., Supakankunti, S. (2018). The impact of cigarette smoking on healthcare utilization among rural residents in China. Journal of Substance Use, 23(6), 626-633. doi: 10.1080/14659891.2018.1489004.

Lightwood, J., Glantz, S. A. (2016). Smoking Behavior and Healthcare Expenditure in the United States, 1992-2009: Panel Data Estimates. Plos Medicine, 13(5), e1002020. doi: 10.1371/journal.pmed.1002020.

Lillard, D. R., Onder, Z. (2019). Health information and life-course smoking behavior: evidence from Turkey. European Journal of Health Economics, 20(1), 149-162. doi: 10.1007/s10198-018-0988-9

Lopez-Nicolas, A., Trapero-Bertran, M., Munoz, C., (2018). Smoking, healthrelated quality of life and economic evaluation. European Journal of Health Economics, 195), 747-756. doi: 10.1007/s10198-017-0919-1.

Maciosek, M. V., Xu, X., Butani, A. L., Pechacek, T. F. (2015). Smokingattributable medical expenditures by age, sex, and smoking status estimated using a relative risk approach. Preventive Medicine, 77, 162-167. doi: 10.1016/j.ypmed.2015.05.019

Max, W. (2001). The financial impact of smoking on health-related costs: A review of the literature. American Journal of Health Promotion, 15(5), 321-331. doi: 10.4278/0890-1171-15.5.321.

Miller, V. P., Ernst, C., Collin, F. (1999). Smoking-attributable medical care costs in the USA. Social Science \& Medicine, 48(3), 375-391. doi: 10.1016/S0277-9536(98)00344-X

Muhyedin, S., Csanadi, M., Kollanyi, Z. (2019). Cultural and Social Factors Affecting Cigarette Smoking Habits among Young Educated People in Kurdistan Region / Iraq. Value in Health, 22, S296. doi: 10.1016/j.jval.2019.04.1419.

OECD (2015). Fiscal Sustainability of Health Systems: Bridging Health and Finance Perspectives. OECD Publishing, Paris. Available at: https://www.oecd. org/gov/budgeting/Fiscal-Sustainability-of-Health-Systems-Policy-Brief-ENG.pdf

OECD (2016a). Health at a Glance: Europe 2016 - State of Health in the EU Cycle. OECD Publishing, Paris. Available at: https://www.oecd.org/els/healthsystems/Health-at-a-Glance-Europe-2016-CHARTSET.pdf. 
OECD (2016b), OECD Factbook 2015-2016: Economic, Environmental and Social Statistics. OECD Publishing, Paris, doi: 10.1787/factbook-2015-en.

OECD (2019a). Health expenditure and financing. Available at: https://stats.oecd.org/Index.aspx?DataSetCode=SHA.

OECD (2019b). Non-Medical Determinants of Health. Available at: https://stats.oecd.org/index.aspx?queryid=30127.

Owen, A. J., Maulida, S. B., Zomer, E., Liew, D. (2019). Productivity burden of smoking in Australia: a life table modelling study. Tobacco Control, 28(3), 297-304, doi: 10.1136/tobaccocontrol-2018-054263.

Pinto, M., Bardach, A., Palacios, A., Biz, A., Alcaraz, A., Rodriguez, B., Augustovski, F., Pichon-Riviere, A. (2019). Burden of smoking in Brazil and potential benefit of increasing taxes on cigarettes for the economy and for reducing morbidity and mortality. Cadernos De Saude Publica, 35(8), e00129118. doi: 10.1590/0102-311X00129118.

Polakowska, M., Kaleta, D., Piotrowski, W., Topor-Madry, R., Puch-Walczak, A., Niklas, A., Bielecki, W., Kozakiewicz, K., Pajak, A., Tykarski, A., Zdrojewski, T., Drygas, W. (2017). Tobacco smoking in Poland in the years from 2003 to 2014 Multi-centre National Population Health Examination Survey (WOBASZ). Polish Archives of Internal Medicine - Polskie Archiwum Medycyny Wewnetrznej, 127(2), 91-99. doi: 10.20452/pamw.3896.

Sharma, R. (2018). Health and economic growth: Evidence from dynamic panel data of 143 years. Plos One. doi: 10.1371/journal.pone.0204940.

Strulik, H. (2018). Smoking kills: An economic theory of addiction, health deficit accumulation, and longevity. Journal of Health Economics, 62, 1-12. doi: 10.1016/j.jhealeco.2018.09.001.

Tai, E. W., Guy, G. P., Steele, C. B., Henley, S. J., Gallaway, M. S., Richardson, L. C. (2018). Cost of Tobacco-related Cancer Hospitalizations in the US, 2014 American Journal of Preventive Medicine, 54(4), 591-595.

doi: 10.1016/j.amepre.2017.12.004.

Toader, E., Firtescu, B. N., Oprea, F. (2017). Determinants of health status and public policies implications - lessons for Romania. Transy/vanian Review of Administrative Sciences, 52e, 128-147. doi: 10.24193/tras.52E.8.

WHO (2013). WHO Report on the Global Tobacco Epidemic, 2013: Enforcing bans on tobacco advertising, promotion and sponsorship. Geneva, Switzerland. Available at: http://apps.who.int/iris/ bitstream/10665/85380/1/9789241505871_eng.pdf.

WHO (2019). Tobacco. Available at:

https://www.who.int/en/news-room/fact-sheets/detail/tobacco.

Xu, X., Bishop, E. E., Kennedy, S. M., Simpson, S. A., Pechacek, T. F. (2015). Annual Healthcare Spending Attributable to Cigarette Smoking An Update. American Journal of Preventive Medicine, 48(3), 326-333.

doi: 10.1016/j.amepre.2014.10.012.

Yang, L., Sung, H., Mao, Z., Hu, T., Rao, K. (2011). Economic costs attributable to smoking in China: update and an 8-year comparison, 2000-2008. Tobacco Control, 2044), 266-272. doi: 10.1136/tc.2010.042028. 